\title{
Noonan Syndrome with Multiple Lentigines and PTPN11 Mutation: A Case with Intracerebral Hemorrhage
}

\author{
Eduardo Orrego-González ${ }^{\mathrm{a}} \quad$ Carlos Martin-Restrepo ${ }^{\mathrm{b}}$ \\ Alberto Velez-Van-Meerbeke ${ }^{a}$ \\ aResearch Group in Neurosciences (NEUROS), School of Medicine and Health Sciences, Universidad del Rosario, \\ Bogotá, Colombia; 'bENIUROS Research Group, Center for Research in Genetics and Genomics-CIGGUR, School of \\ Medicine and Health Sciences, Universidad del Rosario, Bogotá, Colombia
}

\section{Established Facts}

- Noonan syndrome with multiple lentigines (NSML), previously known as LEOPARD syndrome, is an autosomal dominant disorder.

- The majority of cases are due to a missense mutation in the PTPN11 gene.

- Neurologic manifestations described in the literature include sensorineural deafness, mental retardation, and seizures.

\section{Novel Insights}

- Intracerebral hemorrhage is a novel neurologic manifestation of NSML not previously reported in the literature.

- A variant of unknown significance, NM_002474.3:c.4285C>G (NP_002465.1:p.Leu1429Val), in the myosin heavy chain 11 (MYH11) gene was also found.

\section{Keywords}

LEOPARD syndrome · Cerebral hemorrhage - Dysmorphic facial features · Missense mutation - Noonan syndrome

\section{Abstract \\ Noonan syndrome with multiple lentigines (NSML), previ- ously known as LEOPARD syndrome, is a rare autosomal dominant disorder with an unknown prevalence. Character-}

(c) 2021 S. Karger AG, Basel

www.karger.com $/ \mathrm{ms}$

Karger ${ }^{\prime}=$ istics of this disease include cutaneous, neurologic, and cardiologic abnormalities. In this case report, we present a 12-year-old girl who was admitted to the emergency department for acute-onset left weakness, unsteady gait, nausea, and vomiting. Her physical exam notably showed left side upper motor neuron signs and dysmetria. CT scan revealed an acute hemorrhage of the right thalamus. Physical exam exhibited several craniofacial dysmorphisms and lentigines. The genetic test revealed a heterozygous missense mutation 
in the protein tyrosine phosphatase non-receptor type 11 (PTPN11) gene and a variant of unknown significance of the MYH11 gene. To the best of our knowledge, this is the first case of a patient with NSML presenting an intracerebral hemorrhage.

(c) 2021 S. Karger AG, Basel

\section{Introduction}

LEOPARD syndrome (LS), now renamed as Noonan syndrome with multiple lentigines (NSML), is a rare autosomal condition with an unknown prevalence [Sarkozy et al., 2008]. LS belongs to a group of related conditions called the RASopathies, including neurofibromatosis 1, Noonan syndrome (NS), cardiofaciocutaneous (CFC), Costello, and Legius syndromes, as well as several cancer conditions [Aoki et al., 2016]. LEOPARD is an acronym that stands for lentigines and dark spots on the skin (L), electrocardiographic conduction defects (E), ocular hypertelorism $(\mathrm{O})$, pulmonary stenosis $(\mathrm{P})$, abnormal genitalia (A), retarded development (R), and deafness (D) [Tartaglia et al., 2011]. Mutations of the PTPN11 (90\%), $R A F 1(<5 \%), B R A F$, and MAP2K1 (<1\%) genes cause this syndrome, accounting for $95 \%$ of the cases, while in $5 \%$ of the cases, genes are unknown [Gelb and Tartaglia, 2015].

NS is a common autosomal dominant disorder with distinctive craniofacial features, congenital cardiac defects, reduced growth, and bleeding disorders [Rauen, 2013]. The most common gene associated with NS is PTPN11 (50\%) [Rauen, 2013]. Several clinical findings of NS overlap those of NSML, like facial anomalies, some congenital heart defects, sensorineural deafness, and growth retardation [Digilio et al., 2002].

The leading causes of pediatric intracerebral hemorrhage (ICH) are arteriovenous malformations, hematologic abnormalities, and brain tumors [Jordan and Hillis, 2007]. We describe the case of a child who fulfills the criteria of NSML, presenting ICH, and with diagnosis confirmed by a recognized mutation of PTPN11.

\section{Case Presentation}

A 12-year-old girl with a history of mild hypotonia and learning difficulties at school presented to the emergency department with acute-onset left weakness, unsteady gait, nausea, and vomiting. On initial examination, her blood pressure was 116/66 mm $\mathrm{Hg}$, heart rate was 76 beats/min, respiratory rate was 14 breaths/ min, her temperature was $36.8^{\circ} \mathrm{C}$, and oxygen saturation was $98 \%$ without oxygen supplementation.
On neurologic examination, she was alert, attentive, and oriented, with no cranial nerve function alterations. However, she showed marked weakness over the body's left side (muscle power of the left proximal/distal: 3/5 in Medical Research Council Grade). Deep tendon reflexes were augmented on her left side $(+++/++++)$, and she exhibited extensor plantar response (Babinski sign). Dysmetria was noticeable on her left side (positive finger-nose-finger test and heel-knee-shin test). The sensory test was normal.

A CT scan revealed a hemorrhagic lesion on the right thalamus (Fig. 1A-C). A further CT-angiography scan and angiography did not show a source of bleeding (arteriovenous malformations or aneurysms). Her laboratory examination revealed a white blood cell count of $8,800 / \mathrm{mm}^{3}$, hemoglobin of $12.5 \mathrm{~g} / \mathrm{dL}$, platelet count of $280,000 / \mathrm{mm}^{3}$, serum creatinine of $0.8 \mathrm{mg} / \mathrm{dL}$, and blood urea nitrogen of $14 \mathrm{mg} / \mathrm{dL}$. The patient had a prothrombin time of 11 (normal range: 11-13) and partial thromboplastin time of 39 (normal range: 25-45). After 10 days of in-hospital stay with conservative management, her symptoms improved, and she was discharged.

Her height was $147 \mathrm{~cm}$ (75th percentile), the intercanthal distance was $32 \mathrm{~mm}$ (75th percentile), and head circumference was $53 \mathrm{~cm}$ (50th percentile). On her physical exam, several craniofacial findings were noted, including cranial and facial asymmetry, curly hair, triangular face shape, prominent forehead, biparietal alopecia, bushy eyebrows, asymmetric eyelids, and small, low-set, posteriorly rotated ears (Fig. 2A). On her body, the patient exhibited multiple lentigines on the neck, trunk, axillar and inguinal regions, and limbs (Fig. 2A, B), as well as a café-au-lait spot (Fig. 2A). The rest of the physical exam was normal.

The initial differential diagnosis included hereditary diseases associated with stroke (e.g., homocystinuria and cerebral autosomal dominant arteriopathy with subcortical infarcts and leukoencephalopathy [CADASIL]), RASopathies (e.g., neurofibromatosis and Legius syndrome), and collagen and vascular fragility disorders (e.g., Ehlers-Danlos syndrome type IV and Marfan syndrome). Hybrid capture-based next-generation sequencing was performed at Genetica Molecular de Colombia laboratories (Bogota, Colombia) to sequence 91 protein-coding genes related to these diseases. A complete list of these genes is shown in Table 1. The genetic test revealed a heterozygous missense mutation in the protein tyrosine phosphatase non-receptor type 11 (PTPN11) gene, NM_002834.5(PTPN11):c.1493G>T (NP_002825.3:p.Arg498Leu). In addition, the myosin heavy chain 11 (MYH11) gene showed a variant of unknown significance, NM_002474.3: c.4285C>G (NP_002465.1:p.Leu1429Val).

The sisters and other relatives of our patient did not exhibit any of the features of NSML. Additionally, the family history did not exhibit genetic diseases, and the genetic test of the parents was normal. The proband suffered from a de novo mutation.

Over the following months, she developed a "thalamic hand." A follow-up brain MRI, 1 year after the event, showed no residual hematoma with a focal area of encephalomalacia, gliosis, and hemosiderin residues (Fig. 1D-F). The patient also underwent a cardiovascular and otorhinolaryngological assessment, including an ECG, echocardiogram, and hearing test. These did not show any abnormalities. The following year, her academic performance decreased, and she repeated the 5th grade. Overall, she showed a good development.
Orrego-González/Martin-Restrepo/VelezVan-Meerbeke 


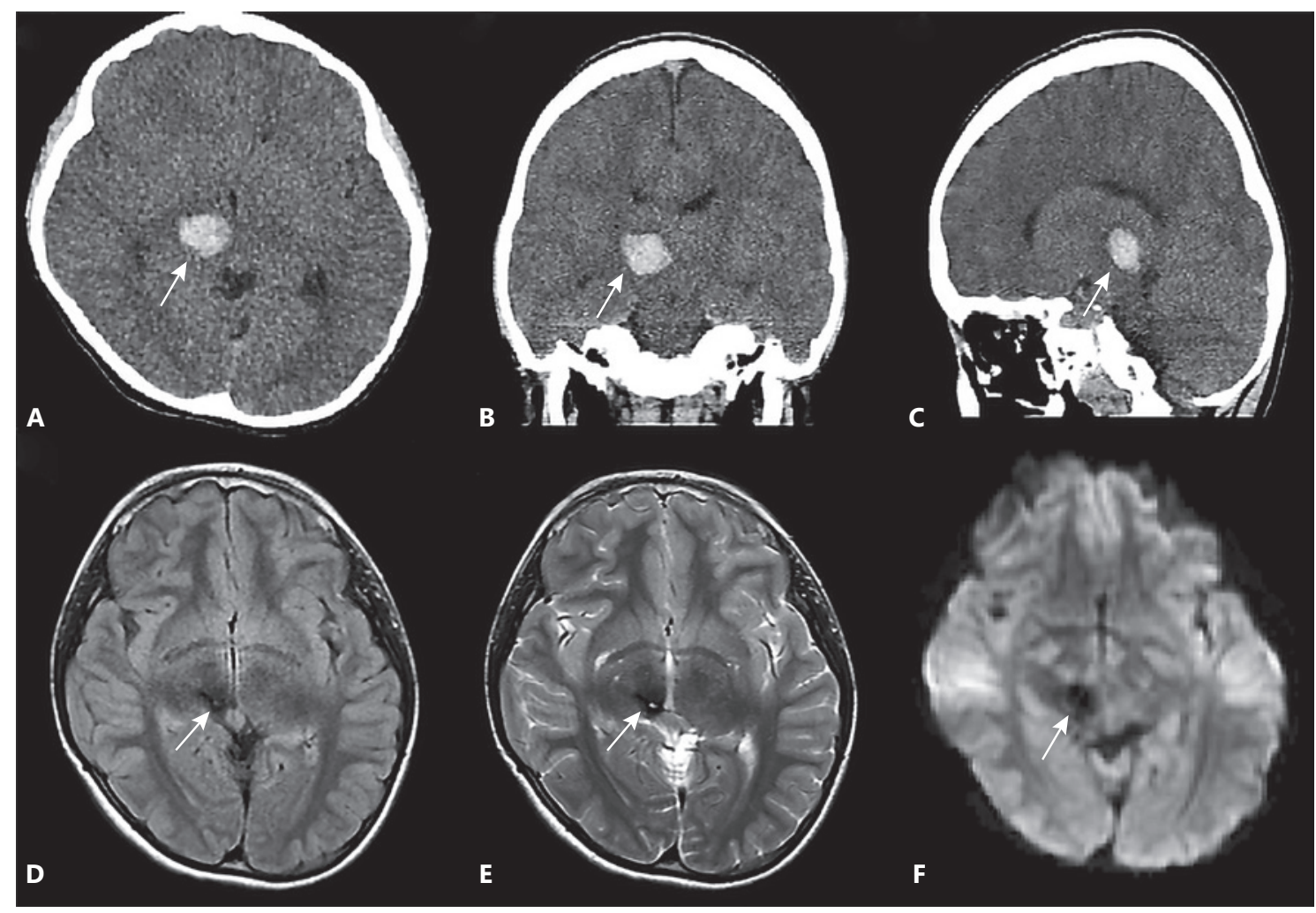

Fig. 1. Computed tomography (CT) on admission (A-C) and follow-up magnetic resonance imaging (MRI) (DF). A Axial CT. B Coronal CT. C Sagittal CT. These images show a hyperdense area (arrows) that compromises the right thalamus. D Axial T2 FLAIR MRI. E Axial T2-weighted image MRI. F Diffusion-weighted image (DWI) MRI. These images demonstrate a hypointense area (white arrows) to gray matter due to the hemosiderin deposition after a year. Signs of encephalomalacia and gliosis are evident. F DWI demonstrated a focal low-intensity area located in the thalami, which is a characteristic of chronic hematomas.

Table 1. List of genes assessed

\begin{tabular}{lllllll}
\hline COL1A1 & COL12A1 & ACTA2 & FBN1 & NOTCH3 & MYH11 & RIN2 \\
COL1A2 & COL4A1 & TGFB1 & FBN2 & MTHFR & SLC2A10 & PLOD3 \\
COL3A1 & COL4A2 & TGFB2 & ENG & PTPN11 & SLC39A13 & PRDM5 \\
COL5A1 & ADAMTS2 & TGFB3 & FV & PECAM1 & SMAD2 & TNXB \\
COL5A2 & PAF & TGFBR1 & F7 & APOE & SMAD3 & PRKG1 \\
COL11A1 & PMF1 & TGFBR2 & F13A & APOH & SMAD4 & PTEN \\
COL11A2 & APP & TGFBR3 & NOTCH1 & ACE & SMARCB1 & RASA1 \\
NF1 & SPRED1 & ATP7A & CCBE1 & CHST14 & SOX18 & MYLK \\
NF2 & ACVRL1 & CBS & CCM2 & CHST3 & STAMBP & NOTCH1 \\
TEK & DSE & EFEMP2 & ELN & FBLN5 & VEGFC & NTM \\
FKBP14 & FLNA & FLNB & FLT4 & FOXC2 & ZNF469 & PDCD10 \\
GATA2 & GDF2 & GJC2 & GLMN & GORAB & IL6 & PLOD1 \\
KIF11 & KRIT1 & LTBP4 & MED12 & SKI & PTPN14 & PYCR1 \\
\hline
\end{tabular}

Genes with mutation and the variant of unknown significance are in bold. PTPN11, missense mutation NM_002834.5:c.1493G>T (NP_002825.3:p.Arg498Leu); MYH11, variant of unknown significance NM_002474.3:c.4285C>G (NP_002465.1:p.Leu1429Val). 

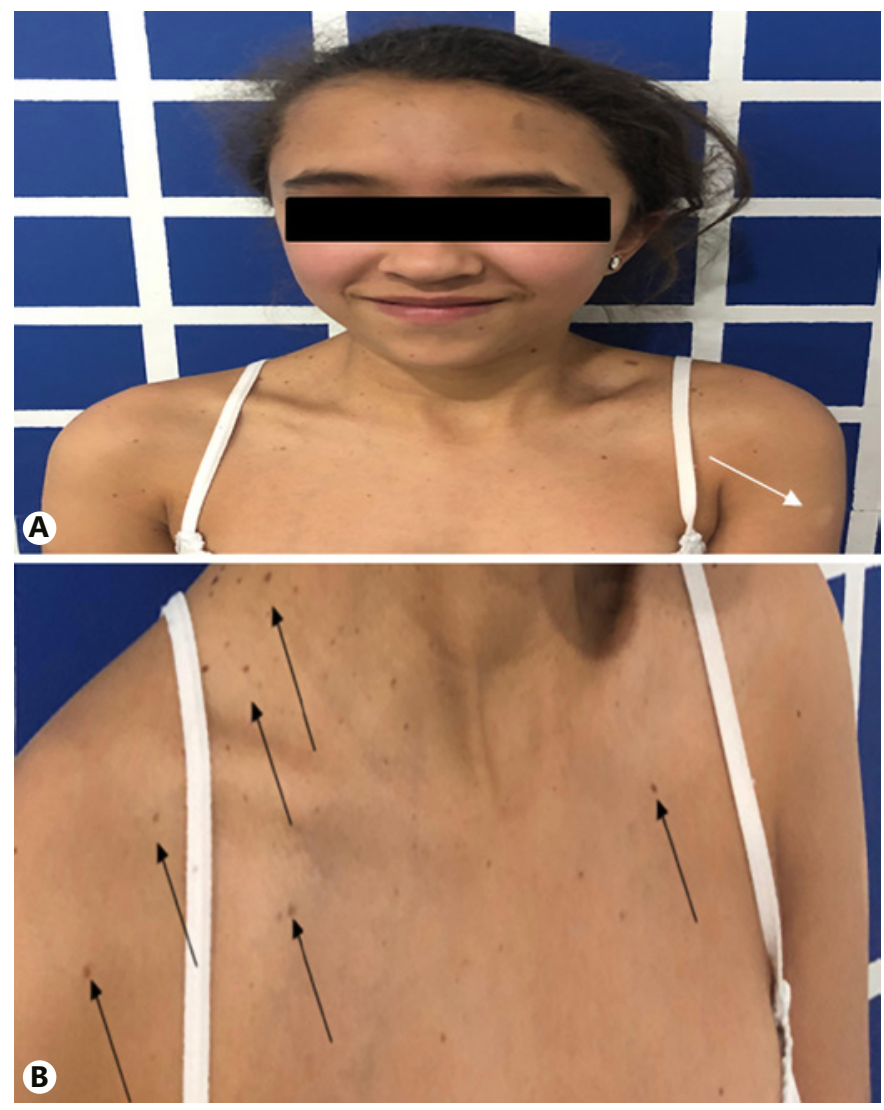

Fig. 2. Physical findings of the patient. A Craniofacial dysmorphisms include cranial and facial asymmetry, curly hair, triangular face shape, prominent forehead, biparietal alopecia, bushy eyebrows, asymmetric eyelids, and small, low-set, posteriorly rotated ears. One café-au-lait spot can be seen on her left arm (white arrow). B Multiple lentigines (black arrows) on the upper torso and neck.

\section{Discussion}

In this case report, we present a 12-year-old female with multiple lentigines who suffered from a hemorrhagic stroke. A heterozygous PTPN11 mutation was found in the girl, leading to the diagnosis of NSML. As described above, this syndrome was previously called LEOPARD syndrome, which is an acronym that stands for its cardinal clinical features. Most patients do not exhibit all these features. To date, diagnosis is based on the recommendations of Voron et al. [1976]: the presence of multiple lentigines and 2 other typical features (cardiac anomalies, ECG abnormalities, genitourinary anomalies, endocrine disorders, neurologic defects, characteristic craniofacial dysmorphism, short stature, skeletal anomalies, and family history) [Yeoh et al., 2014]. Our patient had cutaneous abnormalities (multiple lentigines and café-au-lait spots), craniofacial dysmorphism, and neurologic defects, fulfilling clinical criteria for NSML.

Our patient seemingly might present with an incomplete phenotype of NSML due to the mild neurologic defects presented before the ICH and the lack of cardiac abnormalities. Sarkozy et al. [2004] reported patients with a "partial" phenotype or incomplete clinical spectrum of NSML and attributed this phenomenon to the patients' young age. Some patients in this cohort developed a full-blown phenotype during the following years, including an increase in the number of lentigines and hypertrophic cardiomyopathy [Sarkozy et al., 2004]. Our patient might also present new-onset manifestations, such as sensorineural deafness or cardiac abnormalities, in the forthcoming years. It cannot be ruled out that she will develop new clinical findings in the future. For that reason, we will keep her on close follow-up.

It is well-known that NSML has overlapping clinical features with other RASopathies, and clinical diagnosis can represent a clinical dilemma [Santoro et al., 2014]. This is illustrated in several case reports where an initial diagnosis of one of these syndromes changed later on due to the patients' borderline phenotype [Santoro et al., 2014]. For instance, Carcavilla et al. [2011] reported 3 patients with an initial diagnosis of neurofibromatosis 1 , which in further genetic assays revealed a PTPN11 mutation typical of NSML. Some authors argue as to whether NS and CFC might be the same disorder with a continuum of symptoms or allelic disorders in some patients [Nyström et al., 2008]. These are all reasons that enforce the opinion that the genotype-phenotype relationship is not always straightforward in RASopathies [Santoro et al., 2014]. To solve this, authors like Kavamura et al. [2002] developed a clinical index for CFC with high sensitivity. Others, like Nyström et al. [2008] proposed a classification based on phenotype and genotype (e.g., PTPN11-NS).

In this case, we ruled out 3 RASopathies (neurofibromatosis type 1 , neurofibromatosis type 2 , and Legius syndrome) through genetic testing. NS shares some features with NSML, including facial dysmorphisms, neurologic and cardiac abnormalities. Similar to CFC, it is possible to hypothesize that these are the same disease or allelic disorders for this case in which they share a common mutation. Thus, it is challenging to establish borders between these entities. However, patients with NSML are characterized by a typical dispersed pattern of lentigines (90\%) and the presence of café-au-lait macules [Chinton et al., 2019]. NS has cardinal features such as lymphatic dyspla-
Orrego-González/Martin-Restrepo/VelezVan-Meerbeke 
Table 2. Literature review of the phenotypes of patients with mutation NM_002834.5:c.1493G>T (NP_002825.3:p.Arg498Leu)

\begin{tabular}{|c|c|c|c|c|c|c|c|c|}
\hline Reference & Diagnosis & $\begin{array}{l}\text { Age at diagnosis, } \\
\text { years }\end{array}$ & Skin & $\begin{array}{l}\text { Cardiac } \\
\text { anomalies }\end{array}$ & $\begin{array}{l}\text { Abnormal } \\
\text { ECG }\end{array}$ & $\begin{array}{l}\text { Craniofacial } \\
\text { dysmorphism }\end{array}$ & $\begin{array}{l}\text { Neurologic } \\
\text { defects }\end{array}$ & $\begin{array}{l}\text { Other } \\
\text { features }\end{array}$ \\
\hline $\begin{array}{l}\text { Sarkozy } \\
\text { et al., } 2004\end{array}$ & NSML & 13 & $\begin{array}{l}\text { ML } \\
\text { CALM }\end{array}$ & $\begin{array}{l}\text { Hypertrophic } \\
\text { cardiomyopathy }\end{array}$ & NA & Yes & No & No \\
\hline $\begin{array}{l}\text { Hung } \\
\text { et al., } 2007\end{array}$ & NS & NA & NA & NA & NA & NA & NA & NA \\
\hline $\begin{array}{l}\text { Limongelli } \\
\text { et al., } 2008\end{array}$ & NSML & Fetal & ML & $\begin{array}{l}\text { Aortic regurgitation } \\
\text { Coronary anomalies }\end{array}$ & No & Yes & No & NA \\
\hline
\end{tabular}

NSML, Noonan syndrome with multiple lentigines; NS, Noonan syndrome; ML, multiple lentigines; CALM, café-au-lait macules; NA, not available.

sias, webbing of the neck, pulmonary valve stenosis, and bleeding diathesis [Rauen, 2013]. Our patient did not present these findings; furthermore, the presence of lentigines and a café-au-lait macule make NS less likely in our patient. The genetic test also points to a diagnosis of NSML because mutations in PTPN11 are far more common in this syndrome (90 vs. 50\%) [Chinton et al., 2019]. We believe that future studies should focus on developing a clinical index for diagnosis of NSML to help distinguish it from other RASopathies.

The mutation NM_002834.5(PTPN11):c.1493G > T (NP_002825.3:p.Arg498Leu) has been described in 4 previous patients [Sarkozy et al., 2004; Du-Thanh et al., 2007; Hung et al., 2007; Limongelli et al., 2008]. Their phenotypes are summarized in Table 2. Although the patient reported by Hung et al. [2007] carried the same mutation as our patient, this individual was diagnosed phenotypically as NS. The reports published by Sarkozy et al. [2004] and Du-Thanh et al. [2007] describe patients with NSML presenting this mutation. Facial dysmorphisms were present in all patients, while other symptoms occurred only in some cases, highlighting the presence of ICH as a unique finding of our report.

The PTPN11 gene codes for SHP2, a cytoplasmic protein tyrosine phosphatase (PTP) [Tartaglia et al., 2006]. $\mathrm{Yu}$ et al. [2014] proved that the PTPN11 mutation NM_002834.5(PTPN11):c.1493G>T (NP_002825.3:p. Arg498Leu) diminishes the phosphatase activity of the enzyme, but makes it more prone to be activated by upstream activators (e.g., Gab1). As a consequence, there is a gain of function phenotype and sustained activation of the RAS-ERK1/2 pathway [Yu et al., 2014].

Intracerebral Hemorrhage in Noonan Syndrome with Multiple Lentigines
Some hereditary conditions have been related to stroke; however, the full list of genes associated with stroke remains unknown [Falcone and Woo, 2017]. The major single genetic disorders associated with ischemic stroke include CADASIL, homocystinuria, Marfan syndrome, and Ehlers-Danlos syndrome type IV [Francis et al., 2007]. Patients with CADASIL have a mutation of the $\mathrm{NOTCH} 3$ gene, encoding a transmembrane protein involved in arterial development [Francis et al., 2007]. Classical homocystinuria is caused by a mutation in $C B S$, which codes for the enzyme cystathionine $\beta$-synthase [Francis et al., 2007]. Elevated homocysteine levels are the consequence of this mutation and responsible for the clinical manifestations [Francis et al., 2007].

In Ehlers-Danlos syndrome type IV, mutated collagen (COL3A1) has reduced strength, elasticity, and integrity, while Marfan syndrome is characterized by a mutation of the extracellular matrix protein fibrillin 1 (FBN1), a regulator of metalloproteinases [Francis et al., 2007].

On the other hand, ICH single-gene disorders are familial cerebral amyloid angiopathy (CAA), CADASIL, and COL4A1-related cerebrovascular disease [Rost et al., 2008]. CAA is caused in most cases by a mutation of the $A P P$ gene that leads to a build-up of amyloid in small vessel walls of the cerebral cortex, leptomeninges, and cerebellum [Rost et al., 2008]. COL4A1 mutations are related to autosomal dominant syndromes manifesting with perinatal $\mathrm{ICH}$, adult-onset $\mathrm{ICH}$, microbleeds, lacunar strokes, and leukoaraiosis [Rost et al., 2008]. Other genes are associated with sporadic ICH and could be considered risk factors [Liu et al., 2012]. These include $A P O E(\varepsilon 2$ and $\varepsilon 4$ allelles), $A P O H, E N G, F 7, F 13 A, I L 6$, and TGFBR2 [Liu 
et al., 2012]. All of these discussed genes were assessed through our sequencing (Table 1). As reported, our patient does not exhibit the characteristic features of these disorders, and genetic sequencing ruled out mutations of these genes.

To our knowledge, this is the first report of ICH occurrence in a patient with NSML. PTPN11 mutations have been related to other cerebrovascular diseases (Moyamoya syndrome) [Hung et al., 2011]. This suggests that the phenotype of NSML could be broader. Genotype-phenotype correlation analysis in large samples could help to understand the differences between our patient's findings and previous reports. Variants of unknown significance have insufficient or conflicting evidence regarding the role of molecular alterations in disease [Morales and Hershberger, 2018]. Thus, future research should focus on the structural and mechanistic characterization of this $M Y H 11$ variant to elucidate its pathologic role.

\section{Conclusions}

NSML is a RASopathy with clinical findings that overlap the phenotype of other diseases among this group. Its characteristic features and molecular testing help distinguishing it from other similar conditions. Patients with this particular PTPN11 mutation and ICH as a clinical finding have not been previously reported in the litera- ture. A hemorrhagic stroke should be considered in patients with NSML. The pathogenic role of the MYH11 variant found in this study is still unclear.

\section{Statement of Ethics}

This research was conducted ethically in accordance with the World Medical Association Declaration of Helsinki. Written informed consent was obtained from the patient's legal guardians for publication of this case report and any accompanying images.

\section{Conflict of Interest Statement}

The authors declare that there is no conflict of interest.

\section{Funding Sources}

No funding was received for this research.

\section{Author Contributions}

E.O.-G. and A.V.-V.-M. contributed to the conception and design of the study. A.V.-V.-M. supervised the study. E.O.-G., C.M.R., and A.V.-V.-M. contributed to the acquisition of the patient's data and manuscript drafting, critically revised the article, and reviewed the submitted version of the manuscript. The role of authors was defined according to the guidelines of ICMJE.

\section{References}

Aoki Y, Niihori T, Inoue S, Matsubara Y. Recent advances in RASopathies. J Hum Genet. 2016; 61(1):33-9.

Carcavilla A, Pinto I, Muñoz-Pacheco R, Barrio R, Martin-Frías M, Ezquieta B. LEOPARD syndrome (PTPN11, T468M) in three boys fulfilling neurofibromatosis type 1 clinical criteria. Eur J Pediatr. 2011;170(8):1069-74.

Chinton J, Huckstadt V, Moresco A, Gravina LP, Obregon MG. Clinical and molecular characterization of children with Noonan syndrome and other RASopathies in Argentina. Arch Argent Pediatr. 2019;117(5):330-7.

Digilio MC, Conti E, Sarkozy A, Mingarelli R, Dottorini T, Marino B, et al. Grouping of multiple-lentigines/LEOPARD and Noonan syndromes on the PTPN11 gene. Am J Hum Genet. 2002;71(2):389-94.

Du-Thanh A, Cave H, Bessis D, Puso C, Guilhou JJ, Dereure O. A novel PTPN11 gene mutation in a patient with LEOPARD syndrome. Arch Dermatol. 2007;143(9):1210-1.
Falcone GJ, Woo D. Genetics of Spontaneous Intracerebral Hemorrhage. Stroke. 2017;48: 3420-4.

Francis J, Raghunathan S, Khanna P. The role of genetics in stroke. Postgrad Med J. 2007; 83(983):590-5.

Gelb BD, Tartaglia M. Noonan Syndrome with Multiple Lentigines. In: Adam MP, Ardinger HH, Pagon RA, Wallace SE, Bean LJH, Stephens K, Amemiya A, editors. GeneReviews ${ }^{\circ}$ [Internet]. University of Washington, Seattle; 1993-2020. Initial posting: November 30, 2007; http://www.ncbi.nlm.nih.gov/books/ NBK1383/

Hung CS, Lin JL, Lee YJ, Lin SP, Chao MC, Lo FS. Mutational analysis of PTPN11 gene in Taiwanese children with Noonan syndrome. Formos Med Assoc. 2007;106(2):169-72.

Hung PC, Wang HS, Wong AM. Moyamoya syndrome in a child with Noonan syndrome. Pediatr Neurol. 2011;45(2):129-31.

Jordan LC, Hillis AE. Hemorrhagic Stroke in Children. Pediatr Neurol. 2007;36(2):73-80.
Kavamura MI, Peres CA, Alchorne MM, Brunoni D. CFC index for the diagnosis of cardiofaciocutaneous syndrome. Am J Med Genet. 2002; 112(1):12-6.

Limongelli G, Sarkozy A, Pacileo G, Calabrò P, Digilio MC, Maddaloni V, et al. Genotypephenotype analysis and natural history of left ventricular hypertrophy in LEOPARD syndrome. Am J Med Genet A. 2008;146A(5): 620-8.

Liu B, Zhang L, Yang Q. Genetics of intracerebral hemorrhage: Insights from candidate gene approaches. Neurol India. 2012;60(1):3-8.

Morales A, Hershberger RE. Variants of Uncertain Significance: Should We Revisit How They Are Evaluated and Disclosed?. Circ Genom Precis Med. 2018;11(6):e002169.

Nyström AM, Ekvall S, Berglund E, Björkqvist M, Braathen G, Duchen K, et al. Noonan and cardio-facio-cutaneous syndromes: two clinically and genetically overlapping disorders. J Med Genet. 2008;45(8):500-6.
62

Mol Syndromol 2021;12:57-63

DOI: $10.1159 / 000512374$
Orrego-González/Martin-Restrepo/VelezVan-Meerbeke 
Rauen KA. The RASopathies. Annu Rev Genomics Hum Genet. 2013;14:355-69.

Rost NS, Greenberg SM, Rosand J. The genetic architecture of intracerebral hemorrhage. Stroke. 2008;39(7):2166-73.

Santoro C, Pacileo G, Limongelli G, Scianguetta S, Giugliano T, Piluso G, et al. LEOPARD syndrome: clinical dilemmas in differential diagnosis of RASopathies. BMC Med Genet. 2014; 15:44.

Sarkozy A, Conti E, Digilio MC, Marino B, Morini E, Pacileo G, et al. Clinical and molecular analysis of 30 patients with multiple lentigines LEOPARD syndrome. J Med Genet. 2004; 41(5):e68.
Sarkozy A, Digilio MC, Dallapiccola B. Leopard syndrome. Orphanet J Rare Dis. 2008;3:13.

Tartaglia M, Gelb BD, Zenker M. Noonan syndrome and clinically related disorders. Best Pract Res Clin Endocrinol Metab. 2011;25: 161-79.

Tartaglia M, Martinelli S, Stella L, Bocchinfuso G, Flex E, Cordeddu V, et al. Diversity and functional consequences of germline and somatic PTPN11 mutations in human disease. Am J Hum Genet. 2006;78(2):279-90.
Voron DA, Hatfield HH, Kalkhoff RK. Multiple lentigines syndrome. Case report and review of the literature. Am J Med. 1976;60(3):44756.

Yeoh TY, Wittwer ED, Weingarten TN, Sprung J. Anesthesia and LEOPARD syndrome: a review of forty-nine anesthetic exposures. J Cardiothorac Vasc Anesth. 2014;28(5):1243-50.

Yu ZH, Zhang RY, Walls CD, Chen L, Zhang S, $\mathrm{Wu} \mathrm{L}$, et al. Molecular basis of gain-of-function LEOPARD syndrome-associated SHP2 mutations. Biochemistry. 2014;53(25):413651. 